Article

\title{
Evaluation of the Nutritive Value and the Fatty Acid, Phenol, Tannin and Terpenoid Contents of Nine Pastures in an Alpine District during the Summer Season
}

\author{
Pier Giorgio Peiretti ${ }^{1}\left[\right.$, Sonia Tassone ${ }^{2}$, Narges Vahdani ${ }^{3}$, Giovanna Battelli ${ }^{4}(\mathbb{D}$ and \\ Francesco Gai ${ }^{1, *(\mathbb{D})}$ \\ 1 Institute of Sciences of Food Production, National Research Council, 10095 Grugliasco, Italy; \\ piergiorgio.peiretti@ispa.cnr.it \\ 2 Department of Agriculture, Forestry and Food Sciences, University of Torino, 10095 Grugliasco, Italy; \\ sonia.tassone@unito.it \\ 3 Animal Science Department, Faculty College of Agriculture and Natural Resources, University of Tehran, \\ Karaj, PO Box 31535-1897, Iran; vahdani.narges@gmail.com \\ 4 Institute of Sciences of Food Production, National Research Council, 20133 Milan, Italy; \\ giovanna.battelli@ispa.cnr.it \\ * Correspondence: francesco.gai@ispa.cnr.it; Tel.: +39-011-6709232
}

Received: 8 January 2020; Accepted: 11 February 2020; Published: 13 February 2020

\begin{abstract}
A study was conducted over the summer of 2014 on nine Alpine pastures in the Chisone and Susa Valleys (NW Italy). The aim was to characterize the variation in the chemical composition, gross energy, in vitro true digestibility (IVTD), in vitro neutral detergent fiber digestibility (NDFD), fatty acids (FA), total phenols, total and condensed tannin contents, and terpenoid profile. The dry matter, ash, crude protein, acid detergent fiber, lignin, and gross energy contents of the pastures were found to differ. All the pastures had good IVTD (706-829 g/kg DM) and NDFD (487-694 g/kg $\mathrm{NDF}$ ) values. The most abundant FAs in all the pastures were $\alpha$-linolenic (354-519 $\mathrm{g} / \mathrm{kg}$ of the total FAs), linoleic (75-110 g/kg of the total FAs), and palmitic acid (64-89 $\mathrm{g} / \mathrm{kg}$ of the total FAs) and they differed significantly among pastures. No significant differences were found in the total phenols, or in the total and condensed tannin contents among pastures. Fifty-eight terpenoids were detected and 4-cyclopentene-1, 3-dione, $\beta$-caryophyllene, and eucalyptol were the most abundant. The terpenoids differed both qualitatively and quantitatively among pastures. The results highlight the importance of the great biodiversity of pastures, which provide a balanced distribution of fundamental nutritional elements and bioactive compounds in grasslands.
\end{abstract}

Keywords: chemical composition; digestibility; nutritive value; grassland; bioactive compound

\section{Introduction}

Knowledge of the botanical composition of pastures, in terms of the nutritional quality, the fatty acid (FA) and tannin contents and the terpenoid profile, is a key element in ensuring good coverage of the nutritional needs of livestock and correct classification of the pastures [1].

Several studies have shown that forage species, as well as maturity and environmental conditions, may determine considerable variations in the FA content of pastures [1-3] and forages [4-6]. Alpine pastures have been reported to be rich in phenols, and tannins in particular, and these compounds have been studied because of their potential role in the reduction of ruminant methane production through a modification of ruminal fermentation $[7,8]$. The interest in forage tannins for ruminants is also related 
to their capacity to reduce the loss of native plant FAs, such as $\alpha$-linolenic acid (ALA), during rumen digestion by means of the partial inhibition of ruminal biohydrogenation [9], as observed in both in vitro and in vivo studies $[10,11]$.

Terpenoids are important constituents of the essential oils of plants and are emitted, by plants of different botanical species, as semiochemicals [12]. Moreover, they are more abundant in dicotyledons, such as the Apiaceae and Asteraceae plant families, but are present in lower amounts in some others, like Fabaceae. Monocotyledons are usually poor in these compounds, although, in some cases, they can emit terpenoids as a defense against insects [13].

From a physiological point of view, terpenoids are produced at different levels during the phenological stage of plants, and their production is influenced to a great extent by environmental factors [14]. Moreover, a potential impact of terpenes on milk and dairy products, especially when derived from diverse wild pastures has been shown [15]. A much greater diversity of the terpenes of milk obtained from animals grazing on pasture than of the milk derived from animals reared in confined systems has in fact been suggested [15]. These authors reported that several studies had found that different monoterpenes and sesquiterpenes were able to fully discriminate milk from highland, lowland, pasture, and indoor feeding.

In comparison to indoor feeding substances, mountain pastures are usually rich in plant families characterized by high levels of terpenoids. These strongly scented molecules are transferred directly to the milk fat, and they offer unique characteristics to many protected designations of origin cheeses produced during the grazing season all over the Alps. For this reason, their terpenoid profiles have been proposed as biomarkers to trace mountain cheeses [16]. Mountain pastures are therefore essential for the local economy and land conservation and are of paramount importance for rural sustainable development [17].

To provide valuable support for the natural environment and economic revitalization of local Alpine valley communities, the aim of this research was to characterize the nutritional quality and bioactive compounds of nine Alpine summer pastures sampled from the end of June to early July in the Chisone and Susa Valleys (NW Italy). Fresh grass derived from these grasslands is the prevalent forage resource of the dairy cows in these valleys. These cows are used to produce a typical cheese named "Plaisentif," which is produced during the violet (Viola tricolor) flowering period, this being a mandatory requirement for the product specifications of this typical Alpine cheese.

\section{Materials and Methods}

\subsection{Pasture Sites, Sampling, and Phyto-Pastoral Analysis}

A phyto-pastoral survey was conducted in nine summer pastures located at different altitudes from 1620-2070 m a.s.l. (Table 1), as described by Peiretti et al. [18]. Six of these pastures are located in the Chisone Valley and they have been identified herein with the following abbreviations: A1, A2, A3, A6, A7, and A8, while the other three pastures, A4, A5, and A9, are in the Susa Valley.

Table 1. Sampling date and geolocation of the investigated pastures (A1-A9) during the 2014 summer season.

\begin{tabular}{cccccccccc}
\hline & A1 & A2 & A3 & A4 & A5 & A6 & A7 & A8 & A9 \\
\hline Sampling & $03 / 07 / 2014$ & $30 / 06 / 2014$ & $25 / 06 / 2014$ & $03 / 07 / 2014$ & $25 / 06 / 2014$ & $25 / 06 / 2014$ & $30 / 06 / 2014$ & $30 / 06 / 2014$ & $03 / 07 / 2014$ \\
date & $45^{\circ} 51^{\prime \prime}$ & $45^{\circ} 3^{\prime} 50^{\prime \prime}$ & $44^{\circ} 57^{\prime} 5^{\prime \prime}$ & $44^{\circ} 57^{\prime} 24^{\prime \prime}$ & $44^{\circ} 57^{\prime} 21^{\prime \prime}$ & $44^{\circ} 58^{\prime} 55^{\prime \prime}$ & $44^{\circ} 59^{\prime} 12^{\prime \prime}$ & $45^{\circ} 3^{\prime} 43^{\prime \prime}$ & $44^{\circ} 54^{\prime} 59^{\prime \prime}$ \\
Latitude & $7^{\circ} 7^{\prime} 21^{\prime \prime}$ & $7^{\circ} 2^{\prime} 2^{\prime \prime}$ & $6^{\circ} 57^{\prime} 20^{\prime \prime}$ & $6^{\circ} 48^{\prime} 33^{\prime \prime}$ & $6^{\circ} 50^{\prime} 50^{\prime \prime}$ & $6^{\circ} 55^{\prime} 43^{\prime \prime}$ & $6^{\circ} 54^{\prime} 36^{\prime \prime}$ & $7^{\circ} 2^{\prime} 52^{\prime \prime}$ & $6^{\circ} 53^{\prime} 42^{\prime \prime}$ \\
1867 & 1800 & 1900 & 1870 \\
Longitude & 2040 & 1870 & 1807 & 1620 & 2070 & 1785 & 1867 & 1900 \\
Altitude & & & & & & & & &
\end{tabular}

These pastures are semi-natural grasslands, without any kind of fertilization, with the exception of pasture A4, which was seeded with resistant plants (monocotyledons and Leguminosae) and is used as a ski slope in winter. They were traditionally grazed under rotational grazing systems. None of the 
pastures were ever grazed before the sampling day and, in agreement with the owners of the pastures, were always grazed the day after the sampling.

The botanical composition of the pastures was determined according to the linear analysis method proposed by Daget and Poissonet [19] following the procedure described by Peiretti et al. [18]. The vegetation of the investigated areas was surveyed along $20 \mathrm{~m}$ transects laid out on representative and homogeneous meadows. A metric ribbon was used to trace two transects and an iron rod was inserted into the turf at $50 \mathrm{~cm}$ intervals ( 40 insertions along each transect). The plants in contact with the iron rod were recorded at each insertion.

The chemical analysis sampling was performed using the hand-plucking technique [20]: two separate samples ( $2 \mathrm{~kg}$ each) of herbage were collected in ten points per field above and below the metric ribbon in the transect area, respectively. Plants were cut to a 1-2 cm stubble height, with edging shears, in the morning after evaporation of the dew and were never collected on rainy days. All the samples were immediately refrigerated until the arrival at the laboratory.

The vegetation species were identified by means of the Pignatti dichotomous key [21], and complete results of the phyto-pastoral analysis were reported by Peiretti et al. [18], together with the number of times a plant species was present in a given survey (Species Frequency, SF), the ratio between the SF of a considered species and the sum of the SF of all the species that were present (Species Contribution, SC).

\subsection{Chemical Analysis}

An aliquot of $200 \mathrm{~g}$ was used for each pasture sample, according to the Association of Official Analytical Chemists method [22] to determine the dry matter (DM) content (\#925.40) in duplicate. Another aliquot of $200 \mathrm{~g}$ was immediately refrigerated, freeze-dried, and then brought to room temperature, ground in a Cyclotec mill (Tecator, Herndon, VA, USA) to pass through a 1-mm sieve and stored for qualitative analyses. The freeze-dried samples were analyzed by means of the AOAC methods for total N (\#984.13) and ash (\#923.03) [22]. Neutral detergent fiber (NDFom), and acid detergent fiber (ADFom) were determined as described by Van Soest et al. [23] and expressed exclusive of residual ash, while lignin was determined by solubilization of cellulose with sulfuric acid, as described by Robertson and Van Soest [24], using an Ankom200 Fiber Analyzer (Ankom Technology Corp., Fairport, NY, USA). The NDF of pasture samples was analyzed without sodium sulfite or $\alpha$-amylase. An adiabatic calorimeter bomb (IKA C7000, Staufen, Germany) was used to determine the gross energy (GE) content, while the lipid content was quantified according to the Hara and Radin method [25]. In vitro true digestibility (IVTD) was determined using an Ankom-Daisy incubator (Ankom Technology Corp.) and the in vitro neutral detergent fiber digestibility (NDFD) concentration was subsequently determined using a fiber analyzer (Ankom Technology Corp., Fairport, NY, USA), as previously reported by Peiretti et al. [18]. All the determinations were performed in duplicate.

\subsection{Fatty Acid Analysis}

The FA analysis was performed on a freeze-dried pasture sample $(2 \mathrm{~g})$ according to the method described by Revello Chion et al. [26]. The FA methyl esters (FAME) in hexane were injected into a gas chromatograph (Dani Instruments S.P.A. GC 1000 DPC; Cologno Monzese, Italy) equipped with a flame ionization detector (FID) and a PTV injection port. The separation of the FAME was performed with a Supelcowax-10 fused silica capillary column ( $60 \mathrm{~m}, 0.32 \mathrm{~mm}$ (i.d.), $0.25 \mathrm{~lm}$ ). The peak area was measured using a Dani DDS 1000 Data Station. Each peak was identified according to pure methyl ester standards (Supelco, Bellefonte, PA, USA) and the data were expressed as relative values. The FA composition was expressed as $\mathrm{g} / 100 \mathrm{~g}$ of FA.

\subsection{Phenolic Fraction Determination}

The following phenolic fractions: total phenols (TP), total tannins (TT), and condensed tannins (CT) were determined in the samples. Sample determinations of TP and TT were carried out on the basis of the method described by Makkar et al. [27]. In this way, TP and TT were assessed by means of 
a Folin-Ciocalteu reactive, using the ability of polyvinylpolypyrrolidone to bind tannins and therefore to separate non-tannin phenols from tannin phenols. Both the TP and TT values were expressed as $\mathrm{mg} / \mathrm{kg}$ of DM. The condensed tannins (CT) were analyzed by means of the butanol- $\mathrm{HCl}$-iron method, as described by Porter et al. [28]. CT values were given as leucocyanidin equivalents.

\subsection{Terpenoid Analysis}

Terpenoid analysis was carried out on a freeze-dried pasture sample $(200 \mathrm{mg})$, extracted without the use of solvents, according to the method described by De Noni and Battelli [29], by means of dynamic headspace extraction (Dani Instrument, Cologno Monzese, Italy). Briefly, the extraction conditions were: 5 min equilibrium at $65^{\circ} \mathrm{C}$, purging with $500 \mathrm{ml}$ helium (high-grade purity), adsorption at $40{ }^{\circ} \mathrm{C}$ on a Tenax-TA trap $(270 \mathrm{mg})$, desorption at $280^{\circ} \mathrm{C}$ for $3 \mathrm{~min}$. The obtained data were expressed as arbitrary units, as the peak area of the Total Ion Chromatogram $\times 10^{-6}$.

\subsection{Statistical Analysis}

The variability in the nutritive value, FA, total phenols, and tannin contents of the pastures was analyzed, to establish their statistical significance, by means of an analysis of variance (ANOVA), using the Statistical Package for Social Science [30] to test the effect of pasture. When the values of $F$ were significant $(p<0.05)$, the Ryan-Einot-Gabriel-Welsch range test was used to detect any differences in the means [31]. To examine the relationships between the flora characteristics and the terpenoid profile of the pastures Principal Component Analysis was used [32].

\section{Results}

\subsection{Botanical Composition of the Pastures}

The main forage groups present in the sampled pastures are reported in percentage in Figure 1. The A1 pasture in the Chisone Valley was dominated by Gramineae and Leguminosae (36\% and 20\% of SC, respectively), A2 by Gramineae and Leguminosae ( $40 \%$ and $14 \%$ of SC, respectively), A3 by Gramineae and Leguminosae (both 23\% of SC), A6 by Gramineae and Umbelliferae (20\% and $19 \%$ of SC, respectively), A7 by Asteraceae and Gramineae ( $28 \%$ and $25 \%$ of SC, respectively) and $\mathrm{A} 8$ by Gramineae and Asteraceae ( $34 \%$ and $14 \%$ of SC, respectively). In the Susa Valley pastures, A4 was dominated by Leguminosae and Gramineae ( $25 \%$ and $21 \%$ of SC, respectively), A5 by Gramineae and Asteraceae ( $26 \%$ and $20 \%$ of SC, respectively) and A9 by Leguminosae and Gramineae (18\% and 17\% of SC, respectively).

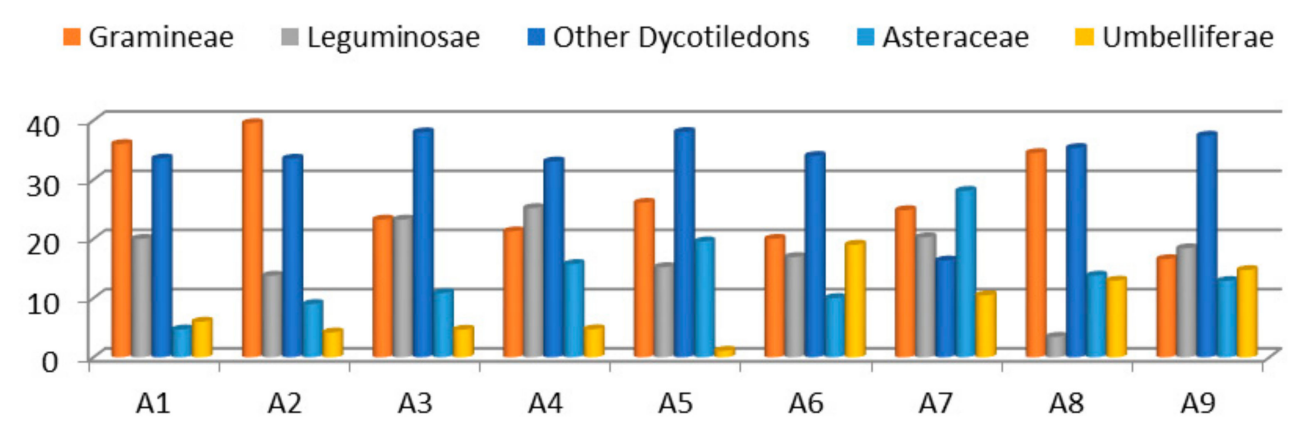

Figure 1. Main forage groups (as the mean percentages of the total number of collected plants) present in the pastures (A1-A9).

Apart from the above-quoted predominant families, there was also a considerable presence of other dicotyledons in some pastures, and in particular: Polygonaceae and Orobanchaceae in A2 (13\% and $7 \%$ of SC, respectively), Rubiaceae and Lamiaceae in A4 (16 and 9\% of SC, respectively), Violaceae in A6 (9\% of SC), Dipsacaceae in A9 (8\% of SC), and Ranunculaceae in A8 (11\% of SC). 


\subsection{Chemical Composition and In Vitro Digestibility}

The chemical composition and in vitro digestibility of the pastures are reported in Table 2. No differences were observed in the lipid and NDFom contents. The mean values were $228 \mathrm{~g} / \mathrm{kg}$ fresh matter for dry matter (DM), $81 \mathrm{~g} / \mathrm{kg}$ DM for ash, $138 \mathrm{~g} / \mathrm{kg}$ DM for crude protein (CP), $343 \mathrm{~g} / \mathrm{kg}$ DM for ADFom, $102 \mathrm{~g} / \mathrm{kg}$ DM for lignin and $17.7 \mathrm{MJ} / \mathrm{kg}$ DM for GE contents in the pastures differed significantly $(p<0.05)$.

Table 2. Chemical composition (g/kg DM basis), gross energy (GE), in vitro true digestibility (IVTD), in vitro neutral detergent fiber digestibility (NDFD) of the pastures (A1-A9).

\begin{tabular}{|c|c|c|c|c|c|c|c|c|c|c|}
\hline & A1 & A2 & A3 & A4 & A5 & A6 & A7 & A8 & A9 & S.E.M. \\
\hline $\mathrm{DM}(\mathrm{g} / \mathrm{kg})$ & $218.8^{\mathrm{ab}}$ & $190.3^{\mathrm{a}}$ & $229.2^{b}$ & $276.8^{c}$ & $207.4^{\mathrm{ab}}$ & $195.3^{\mathrm{ab}}$ & $225.8^{\mathrm{ab}}$ & $231.8^{\mathrm{b}}$ & $273.7^{c}$ & 7.2 \\
\hline Ash & $77.8^{\mathrm{abc}}$ & $75.0^{\mathrm{abc}}$ & $64.7^{\mathrm{ab}}$ & $63.4^{\mathrm{a}}$ & $82.1^{\mathrm{abc}}$ & $106.6^{\mathrm{d}}$ & $94.7^{\mathrm{cd}}$ & $88.0^{\mathrm{bcd}}$ & $74.9^{\mathrm{abc}}$ & 3.3 \\
\hline Crude protein & $158.0^{\mathrm{d}}$ & $150.2^{\mathrm{bcd}}$ & $157.7^{\mathrm{d}}$ & $116.1^{\mathrm{a}}$ & $153.0^{\mathrm{cd}}$ & $130.4^{\mathrm{abc}}$ & $127.4^{\mathrm{ab}}$ & $126.4^{\mathrm{ab}}$ & $122.0^{\mathrm{ab}}$ & 3.5 \\
\hline Lipid & 23.7 & 19.1 & 15.5 & 17.8 & 18.0 & 14.0 & 15.2 & 14.8 & 17.5 & 1.1 \\
\hline NDFom & 562.6 & 587.6 & 534.6 & 575.1 & 509.2 & 515.8 & 522.9 & 551.0 & 523.7 & 8.8 \\
\hline ADFom & $333.5^{\mathrm{a}}$ & $333.4^{\mathrm{a}}$ & $329.7^{a}$ & $403.2^{\mathrm{b}}$ & $325.4^{\mathrm{a}}$ & $349.0^{\mathrm{a}}$ & $338.1^{\mathrm{a}}$ & $337.5^{\mathrm{a}}$ & $339.5^{\mathrm{a}}$ & 6.0 \\
\hline Lignin & $89.7^{\mathrm{ab}}$ & $109.3^{\mathrm{ab}}$ & $117.3^{\mathrm{b}}$ & $98.7^{\mathrm{ab}}$ & $108.4^{\mathrm{ab}}$ & $98.9^{\mathrm{ab}}$ & $79.2^{\mathrm{a}}$ & $103.4^{\mathrm{ab}}$ & $110.4^{\mathrm{b}}$ & 3.0 \\
\hline GE (MJ/kg DM) & $17.5^{\mathrm{a}}$ & $17.8^{\mathrm{a}}$ & $18.5^{\mathrm{b}}$ & $17.6^{\mathrm{a}}$ & $17.6^{\mathrm{a}}$ & $17.6^{\mathrm{a}}$ & $17.5^{\mathrm{a}}$ & $17.6^{\mathrm{a}}$ & $17.8^{\mathrm{a}}$ & 0.1 \\
\hline IVTD (g/kg DM) & 829.3 & 790.3 & 811.8 & 705.7 & 829.1 & 819.8 & 803.4 & 816.5 & 759.9 & 11.1 \\
\hline $\begin{array}{c}\text { NDFD (g/kg } \\
\text { NDF) }\end{array}$ & 694.4 & 643.5 & 648.7 & 487.0 & 664.0 & 650.1 & 621.3 & 669.0 & 540.5 & 19.3 \\
\hline
\end{tabular}

$\mathrm{a}, \mathrm{b}, \mathrm{c}, \mathrm{d}$ Within a row, values with different letters differ $(p<0.05)$; S.E.M., Standard Error Mean.

No differences were observed in the IVTD and NDFD digestibilities. All the pastures are highly digestible, in fact, IVTD ranged between 705 and $829 \mathrm{~g} / \mathrm{kg}$ DM, while NDFD ranged between 487 and $669 \mathrm{~g} / \mathrm{kg}$ NDF.

\subsection{Fatty Acid Profile}

Regarding the FA content, the most abundant FAs in all the pastures were ALA, linoleic acid (LA) and palmitic acid (PA) and they significantly differed among pastures $(p<0.05)$. Some minor FAs (stearic, oleic, and $\gamma$-linolenic acid) overall accounted for 45 to $73 \mathrm{~g} / \mathrm{kg}$ of the total FAs, and they did not differ significantly among pastures (Table 3 ).

Table 3. Fatty acid ( $\mathrm{g} / \mathrm{kg}$ of total FA), total phenols ( $\mathrm{g} / \mathrm{kg} \mathrm{DM})$, total tannin ( $\mathrm{g} / \mathrm{kg} \mathrm{DM})$, and condensed tannin (mg leucocyanidin equivalent/g DM) of the pastures (A1-A9).

\begin{tabular}{|c|c|c|c|c|c|c|c|c|c|c|}
\hline & A1 & A2 & A3 & A4 & A5 & A6 & A7 & A8 & A9 & S.E.M. \\
\hline Palmitic acid & $63.9^{a}$ & $65.9^{\mathrm{ab}}$ & $88.8^{\mathrm{b}}$ & $88.9^{\mathrm{b}}$ & $85.3^{\mathrm{ab}}$ & $86.2^{\mathrm{ab}}$ & $81.9^{\mathrm{ab}}$ & $87.6^{\mathrm{b}}$ & $79.8^{\mathrm{ab}}$ & 2.5 \\
\hline Stearic acid & 16.4 & 16.3 & 26.4 & 23.3 & 21.2 & 24.6 & 23.3 & 26.3 & 33.0 & 1.5 \\
\hline Oleic acid & 11.2 & 14.6 & 18.4 & 17.6 & 13.4 & 10.7 & 11.9 & 16.1 & 19.5 & 1.0 \\
\hline Linoleic acid & $104.0^{\mathrm{bcd}}$ & $104.1^{\mathrm{cd}}$ & $88.5^{\mathrm{ab}}$ & $88.9^{\mathrm{abc}}$ & $104.4^{\mathrm{cd}}$ & $124.7^{\mathrm{e}}$ & $105.0^{\mathrm{cd}}$ & $74.9^{\mathrm{a}}$ & $110.2^{\text {de }}$ & 8.4 \\
\hline$\gamma$-Linolenic acid & 23.8 & 21.5 & 11.5 & 11.8 & 22.3 & 9.8 & 12.7 & 10.7 & 20.9 & 1.6 \\
\hline$\alpha$-Linolenic acid & $354.3^{\mathrm{a}}$ & $395.7^{\mathrm{ab}}$ & $519.1^{c}$ & $503.2^{b c}$ & $470.5^{\mathrm{abc}}$ & $438.3^{\mathrm{abc}}$ & $453.2^{\mathrm{abc}}$ & $518.7^{b c}$ & $420.2^{\mathrm{abc}}$ & 14.3 \\
\hline Total phenols & 36.3 & 44.3 & 61.2 & 49.2 & 53.5 & 52.7 & 37.0 & 42.5 & 52.0 & 2.6 \\
\hline Total tannins & 29.4 & 34.8 & 49.9 & 39.2 & 43.5 & 43.4 & 24.6 & 34.4 & 41.4 & 2.6 \\
\hline $\begin{array}{l}\text { Condensed } \\
\text { tannins }\end{array}$ & 82.6 & 243.1 & 224.3 & 179.7 & 71.6 & 157.1 & 23.9 & 71.6 & 114.6 & 22.7 \\
\hline
\end{tabular}

a,b,c,d,e Within a row, values with different letters differ $(p<0.05)$; S.E.M., Standard Error Mean.

\subsection{Total Phenols, and Total and Condensed Tannins}

No significant differences were found in the total phenols, or in the total and condensed tannin contents among pastures (Table 3). The TP and TT values ranged from 36 to $61 \mathrm{~g} / \mathrm{kg}$ DM and from 25 to $50 \mathrm{~g} / \mathrm{kg}$ DM, respectively. The values of the CT content ranged from 24 to $243 \mathrm{mg}$ leucocyanidin equivalent $/ \mathrm{kg} \mathrm{DM}$, respectively. 


\subsection{Terpenoid Profile}

A total of 58 volatiles, mainly terpenes, were found in the collected pasture samples (Table 4). 4-cyclopentene-1,3-dione, $\beta$-caryophyllene, and eucalyptol were the most abundant terpenes, and they were detected in different concentrations in the pasture samples. Other volatiles $(\delta-3$-carene, allo-ocimene, $\gamma$-curcumene, copaene) were only present in a few locations. Therefore, a great difference in the composition of the volatiles was observed among the pastures. 
Table 4. Terpenoid content range (the min and max data are expressed as arbitrary units of the Total Ion Chromatogram peak areas $\times 10^{-6}$ ) of the pastures (A1-A9).

\begin{tabular}{|c|c|c|c|c|c|c|c|c|c|c|c|c|c|c|c|c|c|c|c|c|c|}
\hline & \multirow{3}{*}{ Compound } & \multirow{3}{*}{$\begin{array}{c}\text { Chemical } \\
\text { Abstract } \\
\text { Service } \\
\text { Number }\end{array}$} & \multirow{3}{*}{$\begin{array}{l}\text { Retention } \\
\text { Index }\end{array}$} & \multicolumn{18}{|c|}{ Pasture } \\
\hline & & & & \multicolumn{2}{|c|}{ A1 } & \multicolumn{2}{|c|}{ A2 } & \multicolumn{2}{|c|}{ A3 } & \multicolumn{2}{|c|}{ A4 } & \multicolumn{2}{|c|}{ A5 } & \multicolumn{2}{|c|}{ A6 } & \multicolumn{2}{|c|}{ A7 } & \multicolumn{2}{|c|}{ A8 } & \multicolumn{2}{|c|}{ A9 } \\
\hline & & & & $\min$ & $\max$ & $\min$ & $\max$ & $\min$ & $\max$ & $\min$ & $\max$ & $\min$ & $\max$ & $\min$ & $\max$ & $\min$ & $\max$ & $\min$ & $\max$ & $\min$ & $\max$ \\
\hline 1 & $\alpha$-pinene & $80-56-8$ & 1023 & 11.68 & 62.69 & 9.78 & 16.42 & 19.78 & 43.31 & 0.73 & 1.22 & 7.15 & 30.51 & 31.83 & 36.61 & 47.84 & 53.41 & 1.50 & 4.96 & 11.80 & 59.07 \\
\hline 2 & camphene & $79-92-5$ & 1065 & 2.11 & 12.20 & 1.40 & 4.68 & 4.62 & 6.29 & 0.13 & 0.20 & 2.24 & 2.54 & 2.16 & 2.81 & 2.67 & 19.97 & 0.20 & 0.85 & 1.44 & 5.32 \\
\hline 3 & L- $\beta$-pinene & $18172-67-3$ & 1105 & 3.82 & 5.85 & 1.47 & 1.76 & 2.83 & 7.84 & 1.29 & 1.77 & 7.64 & 10.54 & 1.96 & 2.19 & 6.66 & 8.76 & 0.27 & 0.55 & 0.39 & 3.40 \\
\hline 4 & $\beta$-pinene & $127-91-3$ & 1113 & 11.66 & 15.08 & 8.26 & 11.02 & 13.60 & 41.18 & 3.26 & 5.60 & 23.93 & 34.14 & 4.77 & 6.83 & 23.57 & 41.29 & 0.34 & 1.46 & 1.92 & 24.94 \\
\hline 5 & unidentified & & 1125 & - & - & 0.65 & 3.40 & 1.66 & 6.86 & - & - & - & 6.29 & - & - & - & - & - & - & - & - \\
\hline 6 & $\delta$-3-carene & $13466-78-9$ & 1125 & - & - & - & - & - & - & - & - & - & 1.41 & 8.37 & 22.36 & - & - & - & - & 5.26 & 8.17 \\
\hline 7 & sabinene & $3387-41-5$ & 1142 & 7.61 & 10.09 & - & - & - & - & - & - & - & - & - & - & 10.55 & 14.87 & - & - & - & - \\
\hline 8 & $\beta$-myrcene & $123-35-3$ & 1166 & 32.75 & 89.77 & - & - & - & 23.72 & 1.25 & 2.15 & - & 1.10 & 15.79 & 36.34 & 7.16 & 16.81 & - & - & - & 5.84 \\
\hline 9 & $\alpha$-terpinene & 99-86-5 & 1185 & - & 5.24 & 1.46 & 2.46 & - & 7.67 & - & - & 1.40 & 5.58 & 8.75 & 20.01 & 13.53 & 15.37 & - & - & 7.06 & 10.74 \\
\hline 10 & limonene & $5989-27-5$ & 1204 & 12.79 & 41.70 & 9.54 & 69.15 & 9.10 & 24.29 & 1.31 & 3.24 & 7.63 & 10.62 & 26.45 & 31.16 & 31.42 & 37.96 & - & 1.13 & 7.96 & 10.28 \\
\hline 11 & eucalyptol & $470-82-6$ & 1217 & 14.95 & 15.35 & 12.15 & 35.07 & 44.98 & 96.58 & 2.91 & 4.01 & 12.47 & 25.88 & 12.50 & 38.89 & 149.37 & 244.43 & 2.13 & 5.71 & 28.75 & 47.22 \\
\hline 12 & $\beta$-ocymene & $3779-61-1$ & 1237 & 4.23 & 16.92 & - & 0.60 & 1.23 & 1.65 & - & - & 0.53 & 0.59 & 2.85 & 4.91 & 3.01 & 4.87 & - & 0.16 & 0.58 & 2.23 \\
\hline 13 & $\gamma$-terpinene & 99-85-4 & 1252 & 13.02 & 15.83 & 3.98 & 5.32 & 5.77 & 23.11 & 1.57 & 1.62 & 8.38 & 8.80 & 27.02 & 44.46 & 27.55 & 31.98 & 0.90 & 3.24 & 13.90 & 50.86 \\
\hline 14 & $o$-cymene & $527-84-4$ & 1278 & 8.63 & 11.69 & 1.53 & 3.44 & 3.48 & 30.25 & 0.29 & 0.34 & 2.25 & 20.50 & 13.34 & 14.23 & 32.29 & 44.32 & 1.01 & 1.17 & 19.29 & 91.13 \\
\hline 15 & terpinolene & $586-62-9$ & 1290 & 20.01 & 21.42 & 3.33 & 4.35 & - & 11.12 & - & 1.85 & 1.79 & 2.50 & 22.57 & 27.89 & 4.90 & 13.61 & 1.15 & 3.11 & 2.01 & 2.75 \\
\hline 16 & allo-ocimene & $3016-19-1$ & 1377 & 5.48 & 43.02 & 0.25 & 0.29 & 0.68 & 1.13 & 0.21 & 0.25 & 0.42 & 0.68 & 6.65 & 13.22 & 3.53 & 5.70 & - & 0.34 & 0.65 & 1.62 \\
\hline 17 & matsutakeol & $3391-86-4$ & 1453 & 33.93 & 33.81 & 22.57 & 29.28 & 29.02 & 35.29 & 18.85 & 18.96 & 26.71 & 33.77 & 14.66 & 22.77 & 27.24 & 42.86 & 12.88 & 44.92 & 19.39 & 32.08 \\
\hline 18 & t-chrysanthenol & $38043-83-3$ & 1491 & 1.19 & 1.44 & 0.00 & 0.60 & 0.71 & 0.94 & 0.37 & 0.51 & 0.68 & 0.70 & 3.70 & 11.38 & 11.77 & 25.66 & - & 0.52 & 1.06 & 5.80 \\
\hline 19 & $\alpha$-copaene & $1000360-33-0$ & 1511 & 5.19 & 21.05 & 2.38 & 4.98 & 5.91 & 8.83 & 1.89 & 5.79 & 2.00 & 8.94 & 7.74 & 11.61 & 13.72 & 26.81 & 0.36 & 2.52 & 5.25 & 6.35 \\
\hline 20 & (t.t)3.5-octadien-2-one & $30086-02-3$ & 1531 & 4.41 & 8.39 & 2.71 & 3.69 & 2.32 & 2.45 & 1.25 & 1.65 & 1.22 & 1.37 & 2.08 & 4.84 & 4.80 & 5.17 & 1.15 & 3.33 & 1.66 & 1.71 \\
\hline 21 & t-chrisanthenyl acetate & 50764-55-1 & 1543 & 5.33 & 13.01 & 0.79 & 2.75 & 10.90 & 12.97 & 2.04 & 2.23 & 1.94 & 2.01 & 7.82 & 8.71 & 3.34 & 35.89 & 1.63 & 4.19 & - & - \\
\hline 22 & 2-bornanone & $464-49-3$ & 1546 & - & - & - & 15.39 & - & - & - & - & - & - & - & - & - & 147.47 & - & 1.66 & 17.71 & 23.85 \\
\hline 23 & linalyl acetate & 115-95-7 & & 4.51 & 6.58 & 0.47 & 2.33 & - & 3.69 & - & 0.35 & 1.62 & 2.46 & 1.47 & 2.93 & 10.25 & 72.83 & - & 0.44 & 4.06 & 9.00 \\
\hline 24 & calarene & $17334-55-3$ & 1558 & 2.07 & 9.92 & 1.08 & 2.44 & 2.26 & 9.05 & - & - & 0.30 & 1.42 & 2.16 & 6.50 & 6.92 & 11.66 & - & 0.82 & 1.76 & 2.36 \\
\hline 25 & 4-cyclopentene-1.3-dione & $930-60-9$ & & 80.59 & 98.18 & 44.94 & 60.05 & 55.14 & 237.28 & 10.20 & 10.26 & 1.03 & 1.77 & 3.48 & 25.74 & 69.11 & 138.91 & 53.70 & 210.23 & 56.52 & 104.18 \\
\hline 26 & $\beta$-elemene & $33880-83-0$ & 1611 & - & 14.53 & - & 11.69 & - & - & - & 1.71 & - & - & 3.90 & 8.31 & - & - & - & - & - & - \\
\hline 27 & isoledene & 95910-36-4 & & 6.10 & 14.20 & 3.62 & 6.13 & - & 16.09 & 0.81 & 3.88 & - & - & 6.38 & 9.37 & 19.79 & 22.50 & 0.67 & 7.48 & 5.40 & 7.92 \\
\hline 28 & $\beta$-caryophyllene & $87-44-5$ & 1623 & 60.02 & 162.31 & 7.39 & 14.12 & 22.45 & 29.32 & 18.19 & 26.57 & 18.51 & 21.09 & 33.38 & 45.29 & 57.46 & 98.28 & 1.62 & 11.40 & 46.77 & 77.63 \\
\hline 29 & cis-calamenene & 72937-55-4 & 1657 & 5.33 & 22.74 & 3.40 & 6.92 & 8.92 & 16.59 & 1.47 & 6.34 & 1.83 & 7.48 & 16.37 & 15.80 & 22.53 & 31.77 & 0.53 & 4.47 & 6.72 & 9.14 \\
\hline 30 & unidentified & & 1662 & 6.72 & 26.76 & 5.71 & 10.87 & 12.54 & 23.45 & 2.05 & 10.37 & 3.02 & 10.46 & 18.55 & 24.52 & 34.92 & 46.62 & 0.73 & 6.07 & 10.54 & 13.90 \\
\hline 31 & $\beta$-farnesene & 28973-97-9 & 1669 & 12.45 & 24.63 & 1.34 & 1.41 & 3.60 & 4.91 & 12.49 & 12.98 & 1.54 & 1.90 & - & 5.98 & 27.50 & 47.94 & 0.20 & 5.35 & 32.06 & 36.47 \\
\hline 32 & $\beta$-sesquiphellandrene & $555-10-2$ & 1684 & 4.64 & 20.59 & 5.91 & 12.77 & - & - & 1.59 & 7.15 & - & - & - & - & - & - & - & - & 7.86 & 10.13 \\
\hline 33 & $\alpha$-elemene & $5951-67-7$ & 1696 & 18.88 & 103.70 & - & - & 16.03 & 30.29 & 3.86 & 13.94 & 3.23 & 11.67 & 21.70 & 37.82 & - & - & 0.62 & 6.94 & 16.05 & 22.03 \\
\hline 34 & unidentified & & 1696 & - & - & 7.54 & 14.47 & - & - & - & - & - & 0.96 & - & - & 45.66 & 64.43 & - & - & - & - \\
\hline 35 & & & 1702 & 2.00 & 13.31 & - & - & - & - & - & - & - & - & - & - & - & - & - & - & - & - \\
\hline 36 & $\delta$-elemene & 20307-84-0 & 1706 & - & - & - & 23.62 & - & - & - & - & - & 13.18 & - & - & - & - & 1.02 & 6.65 & - & - \\
\hline 37 & $\gamma$-muurolene & $30021-74-0$ & 1706 & 6.84 & 26.92 & - & 11.86 & 15.25 & 28.36 & 2.70 & 10.80 & 3.46 & 13.18 & 17.64 & 24.45 & 45.18 & 63.38 & - & - & 12.15 & 15.18 \\
\hline 38 & 10-epi- $\beta$-acoradiene & $28477-64-7$ & 1710 & - & 5.78 & - & - & - & - & - & - & - & - & - & - & - & - & - & - & - & - \\
\hline 39 & viridiflorene & $21747-46-6$ & 1713 & 3.99 & 12.95 & 3.56 & 5.52 & 3.16 & 7.44 & 0.54 & 2.25 & 0.75 & 2.85 & 3.70 & 4.93 & - & 8.79 & - & 1.95 & 4.53 & 5.03 \\
\hline
\end{tabular}


Table 4. Cont.

\begin{tabular}{|c|c|c|c|c|c|c|c|c|c|c|c|c|c|c|c|c|c|c|c|c|c|}
\hline & \multirow{3}{*}{ Compound } & \multirow{3}{*}{$\begin{array}{c}\text { Chemical } \\
\text { Abstract } \\
\text { Service } \\
\text { Number }\end{array}$} & \multirow{3}{*}{$\begin{array}{l}\text { Retention } \\
\text { Index }\end{array}$} & \multicolumn{18}{|c|}{ Pasture } \\
\hline & & & & \multicolumn{2}{|c|}{ A1 } & \multicolumn{2}{|c|}{ A2 } & \multicolumn{2}{|c|}{ A3 } & \multicolumn{2}{|c|}{ A4 } & \multicolumn{2}{|c|}{ A5 } & \multicolumn{2}{|c|}{ A6 } & \multicolumn{2}{|c|}{ A7 } & \multicolumn{2}{|c|}{ A8 } & \multicolumn{2}{|c|}{ A9 } \\
\hline & & & & $\min$ & $\max$ & $\min$ & $\max$ & $\min$ & $\max$ & $\min$ & $\max$ & $\min$ & $\max$ & $\min$ & $\max$ & $\min$ & $\max$ & $\min$ & $\max$ & $\min$ & $\max$ \\
\hline 40 & borneol & 507-70-0 & 1714 & - & 18.58 & - & 0.79 & 1.05 & 2.10 & - & - & 0.19 & - & - & 4.08 & 3.21 & 65.53 & - & - & - & - \\
\hline 41 & epizonarene & $41702-63-0$ & 1723 & 6.34 & 28.50 & 4.55 & 8.76 & 12.76 & 25.96 & 2.84 & 13.32 & 3.01 & 12.44 & 20.77 & 24.57 & 36.68 & 46.20 & - & 4.26 & 11.97 & 14.94 \\
\hline 42 & $\alpha$-muurolene & $10208-80-7$ & 1731 & 7.07 & 30.15 & 4.92 & 9.62 & 11.64 & 29.61 & 1.43 & 7.55 & 2.15 & 8.96 & 13.57 & 18.82 & 43.54 & 51.02 & - & 4.48 & 8.06 & 11.28 \\
\hline 43 & eremophilene & $10219-75-7$ & 1736 & 12.53 & 141.01 & 3.66 & 12.97 & 5.93 & 6.50 & 1.27 & 7.26 & 1.11 & 6.91 & 13.50 & 21.90 & 14.04 & 15.99 & - & 3.13 & 6.34 & 6.90 \\
\hline 44 & carvone & 99-49-0 & 1743 & 2.98 & 5.13 & 1.07 & 21.47 & 2.16 & 2.86 & 0.13 & 12.75 & - & 0.53 & 2.08 & 3.76 & 12.19 & 120.46 & - & 1.53 & 2.06 & 13.90 \\
\hline 45 & 8-cadinene & $483-76-1$ & 1754 & 10.35 & 50.07 & 8.42 & 14.36 & 18.75 & 32.61 & 3.65 & 16.39 & 4.26 & 18.20 & 27.17 & 34.13 & - & 47.20 & - & 7.91 & 15.40 & 19.96 \\
\hline 46 & $\beta$-cadinene & $523-47-7$ & 1755 & - & - & - & - & - & 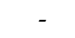 & - & - & - & - & - & - & - & 68.78 & - & - & - & - \\
\hline 47 & germacrene & 28387-44-2 & 1759 & - & - & - & - & 10.91 & 20.96 & - & - & - & 2.52 & - & - & - & - & - & 4.63 & - & - \\
\hline 48 & $\gamma$-cadinene & $39029-41-9$ & 1760 & 11.31 & 29.55 & 4.55 & 8.02 & - & - & 2.22 & 8.95 & - & 10.57 & 13.29 & 18.57 & 27.57 & 40.72 & - & - & 9.43 & 12.44 \\
\hline 49 & copaene & $3856-25-5$ & 1772 & - & 25.00 & - & - & - & - & - & - & 1.41 & 5.33 & - & - & - & - & - & - & - & - \\
\hline 50 & cadinadiene & $16728-99-7$ & 1774 & - & 4.22 & 2.30 & 2.97 & 4.44 & 9.22 & - & 4.02 & - & 5.33 & - & 12.05 & 9.85 & 17.87 & - & - & - & - \\
\hline 51 & selina-3.7(11)-diene & 6813-21-4 & 1775 & - & - & - & 2.10 & - & - & - & - & - & - & - & 17.41 & 3.95 & 10.40 & - & - & - & - \\
\hline 52 & $\alpha$-cadinene & 24406-05-1 & 1780 & 2.01 & 9.02 & - & 3.21 & 4.16 & 7.95 & 0.66 & 3.31 & 0.91 & 3.99 & 6.20 & 8.38 & 10.25 & 15.99 & - & 1.89 & 2.98 & 3.88 \\
\hline 53 & calamenene & $483-77-2$ & 1808 & 3.06 & 10.40 & 2.73 & 4.30 & 6.74 & 10.03 & 2.09 & 6.77 & 2.61 & 6.63 & 8.88 & 9.42 & 11.83 & 14.11 & 1.11 & 2.57 & 5.72 & 6.79 \\
\hline 54 & $\alpha$-patchoulene & $560-32-7$ & 1835 & 1.46 & 8.40 & - & 0.58 & 0.70 & 1.49 & 0.27 & 0.54 & 0.15 & 1.36 & 3.56 & 10.90 & 2.85 & 3.75 & 0.10 & 0.30 & 0.96 & 1.00 \\
\hline 55 & $\alpha$ calacorene & 21391-99-1 & 1869 & 1.29 & 6.44 & 0.85 & 1.76 & 2.40 & 4.22 & 0.78 & 2.29 & 0.66 & 2.30 & 3.01 & 4.99 & 5.11 & 6.26 & 0.19 & 0.94 & 3.98 & 5.20 \\
\hline 56 & thymol & 89-83-8 & 2043 & 0.32 & 0.58 & - & 0.10 & 0.58 & 16.39 & - & 0.31 & 0.16 & 0.45 & 0.59 & 0.63 & 0.60 & 0.70 & - & - & 0.48 & 72.28 \\
\hline 57 & carvacrol & $499-75-2$ & 2070 & 0.15 & 0.20 & - & 0.21 & - & 0.54 & - & 0.12 & - & - & - & - & 0.86 & 1.51 & 0.19 & 0.21 & 0.32 & 3.02 \\
\hline 58 & cadalene & $483-78-3$ & 2102 & 1.33 & 1.34 & 0.57 & 0.73 & 0.74 & 1.16 & 0.18 & 0.65 & 0.27 & 0.55 & 0.64 & 1.18 & 1.64 & 2.09 & 0.80 & 1.30 & 0.72 & 0.87 \\
\hline
\end{tabular}


Regarding the total terpenoids, a huge difference was detected among the pastures, with location A7 being eight-fold richer than the A4 pasture (Figure 2). The relative composition of the volatile organic compounds also differed. Apart from the most abundant terpenoids cited above, the A1 pasture was mainly characterized by eremophilene, $\alpha$-elemene, $\beta$-myrcene, and copaene, and $\gamma$-curcumene and 10 -epi- $\beta$-acoradiene were present only in this location.
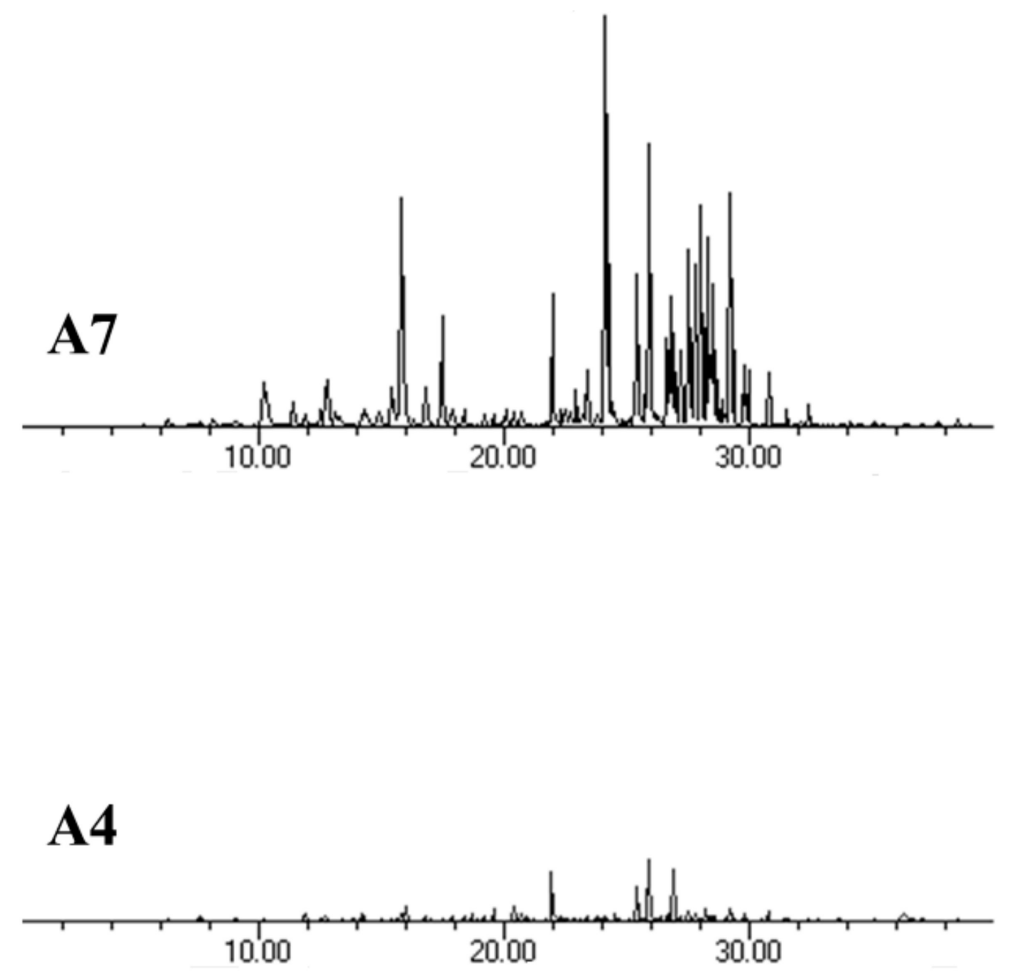

Figure 2. Example of chromatograms of the terpenoid analysis performed according to the method described by De Noni and Battelli [29]. The letters (A7 and A4) refer to the richest and poorest pastures, respectively.

With regard to the other locations, the A2 pasture was mainly characterized by limonene and $\delta$-elemene, the A3 pasture was characterized by eucalyptol and germacrene, the A4 pasture, which showed the lowest level of total terpenoids, was characterized by $\beta$-farnesene, the A5 pasture was characterized by $\beta$-pinene and eucalyptol and the A6 pasture was characterized by $\delta$-3-carene, 3,7(11)-selinadiene, $\gamma$-terpinene, and $\alpha$-pinene. Moreover, almost all the individual terpenoids in the A7 pasture showed the highest concentrations, and it is therefore not surprising that the A7 pasture was the richest location in total volatiles: among these volatiles, the most relevant were eucalyptol, 2-bornanone, carvone, $\gamma$-muurolene, and $\alpha$-pinene.

However, the A8 pasture was as poor as the A4 pasture in total terpenoids and did not show any particular compound, other than the previously cited ones that were present in all the samples. Finally, the A9 pasture was characterized by o-cymene and thymol.

The huge variability in the terpenoid levels was also shown by means of a Principal Component Analysis, which was applied to all the data (Figure 3). The richest pastures (A7 and A1) are visible on the left side of the plot, which is shown in Figure 3, while the poorest (A4 and A8) can be observed on the right side. Interestingly, the two samplings performed in the same pasture showed very different terpenoid profiles. 


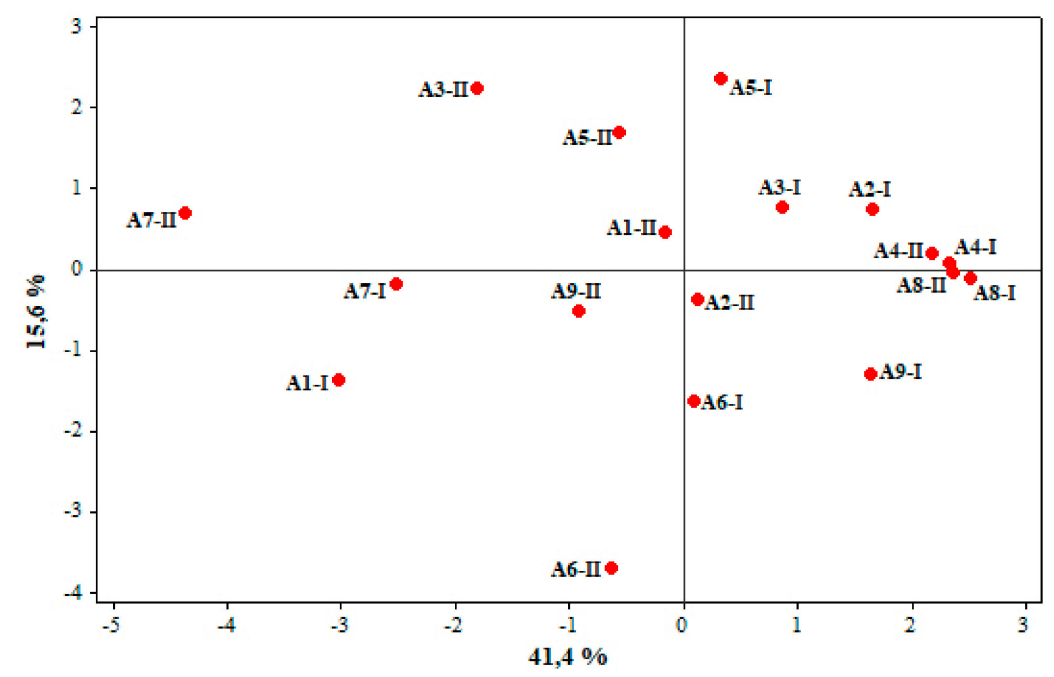

Figure 3. Principal Component Analysis applied to the volatiles identified in the first (I) and second (II) replicates of each pasture (A1-A9).

\section{Discussion}

The results of present study could be useful for dairy farmers in the studied area, and more in general in the Alpine district, to characterize the nutritional quality and bioactive compounds present in the pastures and to correlate them to those of high-quality dairy products, to highlight possible markers capable of linking the product to the production area, and to discriminate these mountain products from those derived from intensive dairy farming.

Overall, the chemical data were in agreement with the average values of all the species reported by Bovolenta et al. [33], even though the here examined pastures are generally more fibrous, as well as less proteic and energetic.

With regards to the fiber fractions and CP content of our study, the NDFom content in the different sampled pastures was similar to those previously reported in a study carried out by Peiretti et al. [1] in the same alpine environment. The ADFom content only differed from the highest recorded content ( $403 \mathrm{~g} / \mathrm{kg} \mathrm{DM})$ in the A4 pasture, which was also the lowest in altitude and resulted to be the pasture with the lowest $\mathrm{CP}$ content $(116 \mathrm{~g} / \mathrm{kg} \mathrm{DM})$. A similar trend between the ADFom and CP contents, which revealed an effect of altitudinal zone, was also observed by Roukos et al. [34], who performed a nutritional quality study on herbage botanical component samples taken from three altitudinal zones (lower, middle, and upper) of a mountainside grassland in North-West Greece. These authors found a $\mathrm{CP}$ content that ranged from 111 to $163 \mathrm{~g} / \mathrm{kg}$ DM, corresponding to an ADFom content ranging from 362 to $277 \mathrm{~g} / \mathrm{kg}$ DM in the lower and upper altitudinal zones, respectively. Different frequencies of Leguminosae at different altitudes can influence the ADFom and CP contents of a pasture [1], and this points out that the altitudinal zone has an important effect on the nutritive value of grasses, legumes, and forbs, as previously reported by Roukos et al. [34].

The NDF digestibility was similar to the values found by Mayer et al. [35] in Alpine wood pastures. The quality and digestibility of forage from grazing lands generally decrease from spring to autumn in all altitudinal zones, as reported by Mountousis et al. [36]. However, we found herbage of good quality in the summer season.

Similar FA profiles to those reported in our study were found by Peiretti et al. [1] in five Alpine pastures during the 2013 grazing period, but these authors found a higher proportion of ALA and LA (their sum ranged from 705 to $734 \mathrm{~g} / \mathrm{kg}$ of the total FAs) than our results (from 458 to $608 \mathrm{~g} / \mathrm{kg}$ of the total FAs). Moreover, these authors only found significant differences for the PA and oleic acid contents between pastures, but not for LA and ALA fatty acids.

The variability of the forage FA profile in the Alpine region in Italy was also studied by Revello Chion et al. [3] between May, when grazing began, to July, when haymaking was performed. They 
found five dominant FAs, and ALA, LA, and PA accounted for more than $850 \mathrm{~g} / \mathrm{kg}$ of the total FAs in both experimental years (2002-2003). During 2002, no significant difference was observed in the LA or PA contents throughout the growing cycle, whereas the ALA significantly decreased from 670 to $407 \mathrm{~g} / \mathrm{kg}$ of the total FAs, while there was no significant change in LA or ALA content throughout the 2003 growing cycle. An FA profile similar to those reported by Revello Chion et al. [3] was found in our study, with the exception of $\gamma$-Linolenic acid and other minor FA contents. Between major FAs, the ALA content of our pastures was similar to those found by Willems et al. [37] in three different alpine pastures, while the LA content was higher than our results.

As far as the TP content of alpine pastures, Willems et al. [37] found a TP content that ranged from 23 to $46 \mathrm{~g} / \mathrm{kg} \mathrm{DM}$ in the swards of the experimental vegetation types. In our study, no significant differences were found in the TP, TT and CT contents between pastures and most of the investigated pastures showed similar or higher TP values than those found by Willems et al. [37] for an alpine vegetation type classified as a highly biodiverse herbaceous-shrub type with a moderate forage quality and high phenolic compound content.

The TP and TT concentrations in our alpine pasture were rather high, compared with those found by Khiaosa-Ard et al. [38] in a similar environment located at an altitude of $2000 \mathrm{~m}$ a.s.l. in the southeast of the Swiss Alps. These authors found that TP and TT ranged from 23 to $30 \mathrm{~g} / \mathrm{kg} \mathrm{DM}$ and from 10 to $17 \mathrm{~g} / \mathrm{kg} \mathrm{DM}$, respectively, and showed that alpine forages were richer in TP and TT than the respective lowland forages. The differences in the tannin contents between our study and those found by Khiaosa-Ard et al. [38] could be related to the different botanical compositions of the Swiss pastures, which were characterized by two main vegetation types, namely Crepido aureae-Festucetum rubrae and Deschampsio cespitosae-Poetum alpinae.

A previous phyto-pastoral analysis carried out in the same sites [18] showed that the most frequent plant species found in the A3 pasture were Onobrychis viciaefolia and Trifolium pratense among the Leguminosae and Dactylis glomerata, Poa alpina and Poa violacea among the Gramineae. Sainfoin (Onobrychis viciaefolia) and red clover (Trifolium pratense) are known to be good sources of CT, with either beneficial or detrimental effects on sustainable ruminant production [39] and the high CT values recorded in the $\mathrm{A} 2$ and $\mathrm{A} 3$ pastures could, therefore, be related to the presence of these plant species. Khiaosa-Ard et al. [38] determined a CT content in pastures located in the southeast of the Swiss Alps that ranged from 0.3 to $3.2 \mathrm{~g} / \mathrm{kg}$ DM but did not find any general anti-bacterial effect of these alpine pastures.

Mountain pastures usually contain a rich variety of terpenoids, due to the great diversity of plant species, which are affected by geographical, agronomic and climatic factors. A rich variety of terpenoids was observed also in this paper as showed by PCA (Figure 3), where the richest and poorest pastures in volatiles are visible on the left and right side of the plot, respectively.

In a similar way, in a mountain environment different from the Alpine ones investigated in our study, a similar richness in the terpenoid profile of the plant species of a mountain pasture located in an eastern region of Northern Spain has been found by Valdivielso et al. [40]. These authors detected more than 75 different individual terpenoids and reported that the total abundance of monoterpenoids was lower than that of sesquiterpenoids in most botanical families and that the most abundant monoterpene in Lamiaceae, Asteraceae, and Ericaceae were $\alpha$-pinene, isoeugenol, $\beta$-thujene, and linalool. Regarding sesquiterpenoids, the most abundant compounds were $\beta$-caryophyllene, $\alpha$-amorphene, and $\alpha$-humulene. Moreover, a higher sesquiterpene/monoterpene ratio was found for the highland forage than for the lowland grazed pasture [41]. Dicotyledon grassland plants generally contain more terpenes than monocotyledons, and the contents can vary widely, according to their botanical family [42]. As a consequence, when natural dicotyledon-rich pastures are fed to dairy cows, the terpene content in their milk and cheese is higher than that of cows fed monospecific forage and concentrates [43].

Fernandez et al. [44] identified six sesquiterpenes that fully discriminated milk produced by dairy cows fed on a highland pasture, a lowland pasture, and an indoor diet. Such a remarkable quantitative 
and qualitative diversity in terpenoids of Alpine pastures should be reflected in the dairy products as a richness in flavor and taste, thus enhancing their global value [45]. These findings were also highlighted by Bozoudi et al. [46], which stated that widespread terpenes in plants could be used as a marker to characterize milk from two mountainous regions of Greece.

\section{Conclusions}

Overall, our research has shown that $\mathrm{DM}$, ash, $\mathrm{CP}, \mathrm{ADF}$, lignin, and GE differed between pastures. All the pastures had good IVTD and NDFD values. The most abundant FAs in all the pastures were $\alpha$-linolenic, linoleic, and palmitic acids and their values differed significantly. No significant differences were found in the total phenols, or in the total and condensed tannin contents between pastures. Fifty-eight terpenoids were detected, and 4-cyclopentene-1, 3-dione, $\beta$-caryophyllene, and eucalyptol were the most abundant. Finally, this research has confirmed the great botanical biodiversity and the good nutritional value of the pastures sampled in the Chisone and Susa Valleys during the summer grazing season.

Author Contributions: Conceptualization, P.G.P. and F.G.; formal analysis, P.G.P., S.T., N.V., G.B., and F.G.; investigation, P.G.P. and F.G.; resources, P.G.P. and F.G.; data curation, P.G.P. and F.G.; writing-original draft preparation, P.G.P., S.T., G.B., and F.G.; writing-review and editing, P.G.P. and F.G.; supervision, P.G.P.; project administration, P.G.P.; funding acquisition, P.G.P. All authors have read and agreed to the published version of the manuscript.

Funding: The work was funded by the Regione Piemonte project: “СССP Caratterizzazione Controllo Certificazione Plaisentif."

Acknowledgments: The authors would like to thank M. Jones for the linguistic revision of the manuscript.

Conflicts of Interest: The authors declare no conflict of interest.

\section{References}

1. Peiretti, P.G.; Gai, F.; Alonzi, S.; Battelli, G.; Tassone, S. Characterisation of Alpine highland pastures located at different altitudes: Forage evaluation, chemical composition, in vitro digestibility, fatty acid and terpene contents. Plant Biosyst. 2017, 151, 50-62. [CrossRef]

2. Leiber, F.; Kreuzer, M.; Nigg, D.; Wettstein, H.R.; Scheder, M.R.L. A study on the cause for the elevated n-3 fatty acids in cow's milk of alpine origin. Lipids 2005, 40, 191-202. [CrossRef] [PubMed]

3. Revello Chion, A.; Tabacco, E.; Peiretti, P.G.; Borreani, G. Variation in the fatty acid composition of Alpine grassland during spring and summer. Agron. J. 2011, 103, 1072-1080. [CrossRef]

4. Dewhurst, R.J.; Scollan, N.D.; Youell, S.J.; Tweed, J.K.S.; Humphreys, M.O. Influence of species, cutting date and cutting interval on the fatty acid composition of grasses. Grass Forage Sci. 2001, 56, 68-74. [CrossRef]

5. Boufaïed, H.; Chouinard, P.Y.; Tremblay, G.F.; Petit, H.V.; Michaud, R.; Belanger, G. Fatty acids in forages. I. Factors affecting concentrations. Can. J. Anim. Sci. 2003, 83, 501-511. [CrossRef]

6. Clapham, W.M.; Foster, J.G.; Neel, J.P.; Fedders, J.M. Fatty acid composition of traditional and novel forages. J. Agric. Food Chem. 2005, 53, 10068-10073. [CrossRef]

7. Jayanegara, A.; Marquardt, S.; Kreuzer, M.; Leiber, F. Nutrient and energy content, in vitro ruminal fermentation characteristics and methanogenic potential of alpine forage plant species during early summer. J. Sci. Food Agric. 2011, 91, 1863-1870. [CrossRef]

8. Morales, R.; Ungerfeld, E.M. Use of tannins to improve fatty acids profile of meat and milk quality in ruminants: A review. Chil. J. Agric. Res. 2015, 75, 239-248. [CrossRef]

9. Jayanegara, A.; Kreuzer, M.; Wina, E.; Leiber, F. Significance of phenolic compounds in tropical forages for the rumen bypass of polyunsaturated fatty acids and the appearance of biohydrogenation intermediates as examined in vitro. Anim. Prod. Sci. 2011, 51, 1127-1136. [CrossRef]

10. Khiaosa-Ard, R.; Bryner, S.F.; Scheeder, M.R.L.; Wettstein, H.R.; Leiber, F.; Kreuzer, M.; Soliva, C.R. Evidence for the inhibition of the terminal step of ruminal $\alpha$-linolenic biohydrogenation by condensed tannins. J. Dairy Sci. 2009, 92, 177-188. [CrossRef]

11. Kälber, T.; Meier, J.S.; Kreuzer, M.; Leiber, F. Flowering catch crops used as forage plants for dairy cows: Influence on fatty acids and tocopherols in milk. J. Dairy Sci. 2011, 94, 1477-1489. [CrossRef] [PubMed] 
12. Petinatti Pavarini, D.; Petinatti Pavarini, S.; Niehues, M.; Peporine Lopes, N. Exogenous influences on plant secondary metabolite levels. Anim. Feed Sci. Technol. 2012, 176, 5-16. [CrossRef]

13. Schnee, C.; Köllner, T.G.; Gershenzon, J.; Degenhardt, G. The maize gene terpene synthase 1 encodes a sesquiterpene synthase catalyzing the formation of $(E)$ - $\beta$-Farnesene, $(E)$-Nerolidol, and $(E, E)$-Farnesol after herbivore damage. Plant Physiol. 2001, 130, 2049-2069. [CrossRef]

14. Verma, N.; Shukla, S. Impact of various factors responsible for fluctuation in plant secondary metabolites. J. Appl. Res. Med. Aromat. Plants 2015, 2, 105-113. [CrossRef]

15. Kilcawley, K.; Faulkner, H.; Clarke, H.; O'Sullivan, M.; Kerry, J. Factors influencing the flavour of bovine milk and cheese from grass based versus non-grass based milk production systems. Foods 2018, 7, 37. [CrossRef] [PubMed]

16. Renna, M.; Cornale, P.; Lussiana, C.; Giordano, M.; Belviso, S.; Zeppa, G.; Battaglini, L.M. Efficacy of fatty acids and terpenoids and weakness of electronic nose response as tracers of Asiago d'Allevo PDO cheese produced in different seasons. Dairy Sci. Technol. 2012, 92, 203-218. [CrossRef]

17. Rainis, S.; Sulli, F.; Rossano, S.; Cividino, S.; Cossio, E. The impact on the landscape, environment and society of new productive chains in a mountain area: Strategies, analysis and future perspectives. J. Agr. Eng. 2012, 43, 3-8. [CrossRef]

18. Peiretti, P.G.; Gai, F.; Alonzi, S.; Tassone, S. Nutritive value and fatty acid profile of birdsfoot trefoil (Lotus corniculatus) and white clover (Trifolium repens) in Alpine pastures. Livest. Res. Rural Develop. 2016, 28, 218.

19. Daget, P.; Poissonet, J. Analyse phytologique des Prairies. In Applications Agronomiques; CNRS-CEPE: Montpellier, France, 1969.

20. Langlands, J.P. Studies on the nutritive value of the diet selected by grazing sheep. VII. A note on hand plucking as a technique for estimating dietary composition. Anim. Prod. 1974, 19, 249-252. [CrossRef]

21. Pignatti, S. Flora d'Italia; Edagricole: Bologna, Italy, 1982.

22. AOAC. Official Methods of Analysis, 15th ed.; Association of Official Analytical Chemists: Arlington, VA, USA, 1990.

23. Van Soest, P.J.; Robertson, J.B.; Lewis, B.A. Methods for dietary fiber, neutral detergent fiber, and nonstarch polysaccharides in relation to animal nutrition. J. Dairy Sci. 1991, 74, 3583-3591. [CrossRef]

24. Robertson, J.B.; Van Soest, P.J. The detergent system of analysis. In The Analysis of Dietary Fibre in Food; James, W.P.T., Theander, O., Eds.; Marcel Dekker: New York, NY, USA, 1981; pp. 123-158.

25. Hara, A.; Radin, N.S. Lipid extraction of tissues with a low-toxicity solvent. Anal. Biochem. 1978, 90, 420-426. [CrossRef]

26. Revello Chion, A.; Tabacco, E.; Giaccone, D.; Peiretti, P.G.; Battelli, G.; Borreani, G. Variation of fatty acid and terpene profiles in mountain milk and "Toma piemontese" cheese as affected by diet composition in different seasons. Food Chem. 2010, 121, 393-399. [CrossRef]

27. Makkar, H.P.S.; Bluemmel, M.; Borowy, N.K.; Becker, K. Gravimetric determination of tannins and their correlations with chemical and protein precipitation methods. J. Sci. Food Agric. 1993, 61, 161-165. [CrossRef]

28. Porter, L.J.; Hrstich, L.N.; Chan, B.G. The conversion of procyanidins and prodelphinidins to cyanidin and delphinidin. Phytochemistry 1986, 25, 223-230. [CrossRef]

29. De Noni, I.; Battelli, G. Terpenes and fatty acid profiles of milk fat and "Bitto" cheese as affected by transhumance of cows on different mountain pastures. Food Chem. 2008, 109, 299-309. [CrossRef] [PubMed]

30. SPSS. Statistical Package for Social Science; Version 11.5.1 for Windows; SPSS Inc.: Chicago, IL, USA, 2002.

31. Hochberg, Y.; Tamhane, A.C. Multiple Comparison Procedures; Wiley: New York, NY, USA, 1987.

32. Piernik, A. Numerical Methods in Ecology, 1st ed.; Scientific Publishers of Nicolaus Copernicus University: Torun, Poland, 2008; p. 92.

33. Bovolenta, S.; Spanghero, M.; Dovier, S.; Orlandi, D.; Clementel, F. Chemical composition and net energy content of alpine pasture species during the grazing season. Anim. Feed Sci. Technol. 2008, 146, 178-191. [CrossRef]

34. Roukos, C.; Papanikolaou, K.; Karalazos, A.; Chatzipanagiotou, A.; Mountousis, I.; Mygdalia, A. Changes in nutritional quality of herbage botanical components on a mountain side grassland in North-West Greece. Anim. Feed Sci. Technol. 2011, 169, 24-34. [CrossRef]

35. Mayer, A.C.; Stöckli, V.; Huovinen, C.; Konold, W.; Estermann, B.L.; Kreuzer, M. Herbage selection by cattle on sub-alpine wood pastures. Forest. Ecol. Manag. 2003, 181, 39-50. [CrossRef] 
36. Mountousis, I.; Papanikolaou, K.; Stanogias, G.; Chatzitheodoridis, F.; Roukos, C. Seasonal variation of chemical composition and dry matter digestibility of rangelands in NW Greece. J. Cent. Eur. Agr. 2008, 9, 547-556.

37. Willems, H.; Kreuze, M.; Leiber, F. Alpha-linolenic and linoleic acid in meat and adipose tissue of grazing lambs differ among alpine pasture types with contrasting plant species and phenolic compound composition. Small Rumin. Res. 2014, 116, 153-164. [CrossRef]

38. Khiaosa-Ard, R.; Soliva, C.R.; Kreuzer, M.; Leiber, F. Effects of species-diverse high-alpine forage on in vitro ruminal fermentation when used as donor cow's feed or directly incubated. Animal 2012, 6, 1764-1773. [CrossRef] [PubMed]

39. Waghorn, G. Beneficial and detrimental effects of dietary condensed tannins for sustainable sheep and goat production-Progress and challenges. Anim. Feed Sci. Technol. 2008, 147, 116-139. [CrossRef]

40. Valdivielso, I.; Bustamante, M.A.; Aldezabal, A.; Amores, G.; Virto, M.; de Gordoa, J.R.; de Renobales, M.; Barron, L.J.R. Case study of a commercial sheep flock under extensive mountain grazing: Pasture derived lipid compounds in milk and cheese. Food Chem. 2016, 197, 622-633. [CrossRef] [PubMed]

41. Engel, E.; Ferlay, A.; Cornu, A.; Chilliard, Y.; Agabriel, C.; Bielicki, G.; Martin, B. Relevance of isotopic and molecular biomarkers for the authentication of milk according to production zone and type of feeding of the cow. J. Agric. Food Chem. 2007, 55, 9099-9108. [CrossRef] [PubMed]

42. Tornambé, G.; Cornu, A.; Pradel, P.; Kondjoyan, N.; Carnat, A.; Petit, M.; Martin, B. Changes in terpene content in milk from pasture-fed cows. J. Dairy Sci. 2006, 89, 2309-2319. [CrossRef]

43. Agabriel, C.; Cornu, A.; Journal, C.; Sibra, C.; Grolier, P.; Martin, B. Tanker milk variability according to farm feeding practices: Vitamins A and E, carotenoids, color, and terpenoids. J. Dairy Sci. 2007, 90, 4884-4896. [CrossRef]

44. Fernandez, C.; Astier, C.; Rock, E.; Coulon, J.B.; Berdagué, J.L. Characterization of milk by analysis of its terpene fractions. Int. J. Food Sci. Technol. 2003, 38, 445-451. [CrossRef]

45. Gai, F.; Battelli, G.; Bottero, M.T.; Civera, T.; Dalmasso, A.; Pattono, D.; Peiretti, P.G. Influence of Alpine highland pasture on the fatty acid and terpene composition of milk and Plaisentif cheese from various Piedmont farms. Options méditerranéennes. Serie A: séminaires méditerranéens 2014, 109, 293-296.

46. Bozoudi, D.; Claps, S.; Abraham, E.M.; Parissi, Z.M.; Litopoulou-Tzanetaki, E. Volatile organic compounds of mountainous plant species and the produced milk as affected by altitude in Greece: A preliminary study. Int. J. Dairy Technol. 2019, 72, 159-164. [CrossRef] 